\title{
THE NATURE OF TRANSITION BLAZARS
}

\author{
J. J. Ruan ${ }^{1}$, S. F. Anderson ${ }^{1}$, R. M. Plotkin ${ }^{2}$, W. N. Brandt ${ }^{3,4}$, T. H. BurnetT ${ }^{5}$, A. D. Myers ${ }^{6}$, And D. P. Schneider ${ }^{3,4}$ \\ ${ }^{1}$ Department of Astronomy, University of Washington, Box 351580, Seattle, WA 98195, USA; jruan@ astro.washington.edu \\ ${ }^{2}$ Department of Astronomy, University of Michigan, 500 Church Street, Ann Arbor, MI 48109, USA \\ ${ }^{3}$ Department of Astronomy \& Astrophysics, 525 Davey Lab, The Pennsylvania State University, University Park, PA 16802, USA \\ ${ }^{4}$ Institute for Gravitation and the Cosmos, The Pennsylvania State University, University Park, PA 16802, USA \\ ${ }^{5}$ Department of Physics, University of Washington, Seattle, WA 98195-1560, USA \\ ${ }^{6}$ Department of Physics and Astronomy 3905, University of Wyoming, 1000 East University, Laramie, WY 82071, USA \\ Received 2014 August 9; accepted 2014 October 6; published 2014 November 20
}

\begin{abstract}
Blazars are classically divided into the BL Lacertae (BLL) and flat-spectrum radio quasar (FSRQ) subclasses, corresponding to radiatively inefficient and efficient accretion regimes, respectively, largely based on the equivalent width (EW) of their optical broad emission lines (BELs). However, EW-based classification criteria are not physically motivated, and a few blazars have previously "transitioned" from one subclass to the other. We present the first systematic search for these transition blazars in a sample of 602 unique pairs of repeat spectra of 354 blazars in the Sloan Digital Sky Survey, finding six clear cases. These transition blazars have bolometric Eddington ratios of $\sim 0.3$ and low-frequency synchrotron peaks, and are thus FSRQ-like. We show that the strong EW variability (up to an unprecedented factor of $>60$ ) is due to swamping of the BELs from variability in jet continuum emission, which is stronger in amplitude and shorter in timescale than typical blazars. Although these transition blazars appear to switch between FSRQ and BLL according to the phenomenologically based EW scheme, we show that they are most likely rare cases of FSRQs with radiatively efficient accretion flows and especially strongly beamed jets. These results have implications for the decrease of the apparent BLL population at high redshifts, and may lend credence to claims of a negative BLL redshift evolution.
\end{abstract}

Key words: BL Lacertae objects: general - galaxies: active - quasars: emission lines - quasars: general

Online-only material: color figures

\section{INTRODUCTION}

Blazars are a relatively rare class of active galactic nuclei (AGNs) in which a jet is aligned closely along the observed line of sight (Blandford \& Rees 1978). These jets are relativistically beamed, leading to unique multi-wavelength properties from the radio through $\gamma$-rays (Antonucci 1993; Urry \& Padovani 1995). Classically, blazars have been divided into the flat-spectrum radio quasar (FSRQ) and BL Lacertae (BLL) subclasses based on whether any optical broad emission lines (BELs) have restframe equivalent widths (EWs) above (in the case of FSRQs) or below (in the case of BLLs) $5 \AA$ (e.g., Stickel et al. 1991; Stocke et al. 1991; Perlman et al. 1996; Plotkin et al. 2010). These two subclasses of blazars also show systematic differences in Eddington ratios (Dai et al. 2007), $\gamma$-ray properties (Ackermann et al. 2011), and the luminosities and frequencies of the synchrotron and inverse-Compton peaks of their spectral energy distributions (SEDs; Padovani \& Giommi 1995; Sambruna et al. 1996; Fossati et al. 1998; Abdo et al. 2010a), among many other differences.

The physical distinction between FSRQs and BLLs is suspected to stem from the divergent natures of their underlying accretion flows around the central supermassive black holes (Maraschi \& Tavecchio 2003; Ghisellini \& Tavecchio 2008). FSRQs are believed to harbor geometrically thin, optically thick accretion disks that are accreting rapidly, with Eddington ratios $\gtrsim 0.1$, similar to (and perhaps systematically higher than) typical Type 1 quasars (Shaw et al. 2012). The existence of BELs in their optical spectra from high-velocity gas clouds, as well as strong emission in the infrared from dusty torii, are well described by the standard radio-loud unification scheme of AGNs (Urry \& Padovani 1995). In contrast, BLLs are thought to have geometrically thick, optically thin accretion disks (at least in their inner regions) where the accretion is radiatively inefficient (Narayan \& Yi 1994), with Eddington ratios generally $\lesssim 0.01$ (Wang et al. 2002; Dai et al. 2007). Their lack of BELs and dust emission suggests that the properties of the accretion flow and the structure of the surrounding gas in BLLs are physically different from FSRQs.

The classical EW-based classification of blazars between FSRQs and BLLs is not physically motivated, and a handful of blazars have previously been reported to transition between these two classes in repeat spectroscopic observations due to variability in their BEL EWs (e.g., Ghisellini et al. 2011; Shaw et al. 2012). These rare "transition" blazars ironically include the namesake BL Lacertae object, in which the rest-frame $\mathrm{H} \alpha$ EW has been reported to vary between approximately 0 to $\sim 7 \AA$ (Vermeulen et al. 1995; Corbett et al. 1996). Analysis of longterm spectroscopic monitoring of BL Lacertae by Corbett et al. (2000) demonstrated that this variability in the BEL EW is anticorrelated with the continuum luminosity, suggesting that the strongly variable jet continuum emission is swamping the BEL flux, leading to the transition blazar phenomena. More recent reports of Fermi-detected transition blazars by Shaw et al. (2012) have provided observational motivation for investigations into the mysterious nature of these objects (see also Isler et al. 2013). Ghisellini et al. (2011) showed that many Fermi-detected BLLs with $\gamma$-ray properties similar to FSRQs also have SEDs and BEL luminosities that are FSRQ-like, thus suggesting that some BLLs may actually be FSRQs with particularly strong jet continuum emission. Based on their findings, Ghisellini et al. (2011) also suggested an improved classification scheme based on the Eddington ratio calculated from the BEL luminosities; this approach is more physically motivated and particularly 
helpful for transition blazars (also see Giommi et al. 2012; Sbarrato et al. 2012).

Correct classification of transition blazars is critical not only for studies of the different properties of FSRQs and BLLs (e.g., along the "blazar sequence"; Fossati et al. 1998), but also has important implications for their redshift evolution. For example, BLLs are more commonly found at lower redshifts, leading to many reports of apparent negative redshift evolution (Maccacaro et al. 1984; Wolter et al. 1994; Rector et al. 2000; Beckmann et al. 2003), although this interpretation has often been disputed (Caccianiga et al. 2002; Padovani et al. 2007; Marchã \& Caccianiga 2013). Misclassifications of blazars can artificially lead to an apparent lack of high-redshift BLLs, significantly affecting evolutionary scenarios of the blazar sequence (e.g., Böttcher \& Dermer 2002) and the blazar contributions to the cosmic $\gamma$-ray background (e.g., Abdo et al. 2010b). These issues of correct classification face the further challenge of obtaining correct redshifts from the often featureless spectra of blazars; strong BEL EW variability in transition blazars can be used to determine or constrain redshifts in repeat spectra during epochs in which the EWs are large (Shaw et al. 2013a).

In this paper, we present the first systematic search for transition blazars in a large sample of repeat spectra of known blazars in the Sloan Digital Sky Survey (SDSS; York et al. 2000). Many previous reports of transition blazars have been based on comparison to EW classifications in the literature; the homogeneous nature of SDSS spectra will allow a more robust measurement of their variable spectral properties over timescales of over a decade in the observed frame. To complement the repeat optical spectra in our study, we also adopt a multi-wavelength approach to investigate the $\gamma$-ray, optical variability, and SED properties of these rare objects, tying our findings to the many recent investigations of blazar properties in these domains.

The outline of this paper is as follows. In Section 2, we present our sample of repeat blazar spectra and spectral analysis to identify transition blazars. In Section 3, we discuss the spectral and multi-wavelength properties of the transition blazars in our sample. In Section 4, we comment on the individual transition blazars in our sample. In Section 5, we discuss the nature of these transition blazars based on their properties. We summarize and conclude in Section 6. Throughout the paper, EW refers to the EW calculated in the rest-frame. We assume a standard $\Lambda \mathrm{CDM}$ cosmology with $\Omega_{M}=0.3, \Omega_{\Lambda}=0.7$, and $H_{0}=70 \mathrm{~km} \mathrm{~s}^{-1} \mathrm{Mpc}^{-1}$, consistent with the WMAP nine year results of Hinshaw et al. (2013).

\section{DATA AND SEARCH FOR TRANSITION BLAZARS}

\subsection{Analysis of SDSS Spectra}

Our data are taken from the SDSS-III (Gunn et al. 2006; Eisenstein et al. 2011) Data Release 10 (DR10; Ahn et al. 2014), which is publicly available and also includes all data from the SDSS-I/II portions of the survey. DR10 includes $\sim 4 \times 10^{6}$ optical spectra over approximately $14,000 \mathrm{deg}^{2}$ of sky, including $\sim 1.6 \times 10^{5}$ quasars (Pâris et al. 2014), selected primarily from the imaging portion of the survey in a variety of science programs (Ross et al. 2012). In our investigation, we include all optical spectra taken from the primary survey portion of SDSSI/II on the SDSS spectrograph, as well as spectra from the BOSS spectrograph used in SDSS-III (spectral resolution $R \sim 2000$ ). Due to differences between the spectrographs, fiber system, and spectral reduction pipeline between these phases of the SDSS survey (see Bolton et al. 2012; Smee et al. 2013), some small

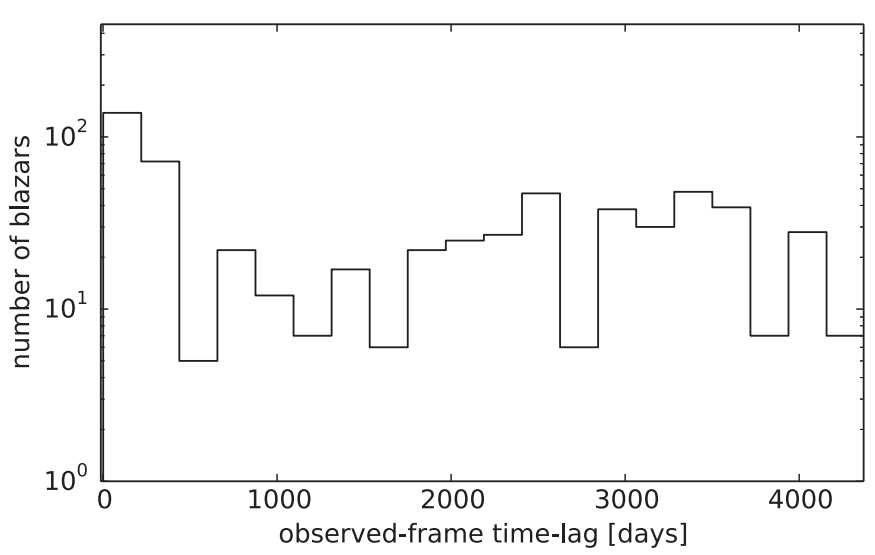

Figure 1. Histogram of the observed-frame time lags between each pair of repeat blazar spectra in our sample of 602 unique pairs. The median redshift of blazars with measured redshifts is 1.05 , although this value is strongly biased toward FSRQs, which have systematically higher redshifts than BLLs.

differences in absolute flux calibration may be present in spectra taken at different epochs (Dawson et al. 2013). These differences do not strongly affect our results, which are primarily based on spectral properties which are normalized to the continuum (e.g., EWs).

Occasionally, repeat spectra of the same objects in SDSS were taken, either due to low signal-to-noise ratios in the first epoch or as part of the survey plan. To create a sample of known blazars with repeat spectra in SDSS-III, we match all objects with repeat spectra available in DR10 to the 3149 blazars in the ROMABZCAT $^{7}$ catalog of Massaro et al. (2009). This comparison results in a sample of 354 blazars with repeat observations, including a few with more than two epochs. Since the classical EW-based classification is based on whether any BELs in the observed optical spectrum are above or below $5 \AA$, we will compare the BEL EWs in pairs of repeat spectra of each blazar to identify cases where the BEL crossed this EW threshold. For blazars with more than two epochs of spectra, we will compare all unique pairs, leading to a total of 602 unique pairs of repeat spectra of the 354 blazars. Blazars are known to be highly variable even in intra-night observations, and the timescales on which blazars can transition between the FSRQ and BLL subclasses is not known; we thus place no cuts on the time lag between repeat observations for our sample, which range from intra-night to approximately $12.5 \mathrm{yr}$ in the observed frame. The distribution of time lags for our sample is shown in Figure 1.

We correct all repeat blazar spectra in our sample for Galactic extinction, using the maps of Schlegel et al. (1998) and the Milky Way reddening law of Cardelli et al. (1989). We also shift the repeat spectra of each blazar into the rest frame using redshifts provided by the ROMA-BZCAT catalog, aware of the fact that the redshifts of many blazars in this cata$\log$ are missing, uncertain, or occasionally incorrect. To ensure that these redshifts we use are not obviously incorrect, we visually inspect all 602 unique pairs of repeat blazar spectra in our sample. In the visual inspection, we mask pixels in each spectrum with SDSS flags set for NOPLUG, BADTRACE, BADFLAG, BADARC, MANYBADCOLUMNS, MANYREJECTED, NEARBADPIXEL, LOWFLAT, FULLREJECT, SCATTEREDLIGHT, NOSKY, BRIGHTSKY, COMBINEREJ, and REDMONSTER (for details on these flags, see Stoughton et al. 2002). Due to this spectrum quality mask, 16

\footnotetext{
7 http://www.asdc.asi.it/bzcat/
} 
of the 602 unique pairs of repeat blazar spectra in our sample were removed.

As expected, our visual inspection reveals that the overwhelming majority of blazars appear to have spectra that are either featureless (canonical BLLs) or have moderately strong BELs (canonical FSRQs). Some outliers include BLLs that possessed strong host-galaxy contributions (e.g., misaligned BLLs), blazars with intrinsic and line-of-sight absorption features, as well as a small handful in which the emission lines strongly varied in EW between the different spectral epochs. To quantitatively identify blazars that have transitioned from FSRQs to BLLs (or vice versa) in our sample, we measure the properties of the $\mathrm{CIV}_{\mathrm{IV}} \lambda 1546, \mathrm{C}$ III] $\lambda 1906, \mathrm{Mg}$ II $\lambda 2800$, and $\mathrm{H} \alpha \lambda 6565$ BELs (in vacuums wavelengths) where possible.

\subsection{BEL Fitting and the Transition Blazar Sample}

The details of our BEL fitting closely follow Shen et al. (2011, hereafter SH11) to allow for comparison of the BEL properties of our transition blazars to the general SDSS quasar population. The primary difference in our fitting in comparison to $\mathrm{SH} 11$ is that we opt not to fit templates for $\mathrm{Fe}$ emission since the continua in transition blazars are strongly dominated by jet emission, and thus the Fe line complexes are not well detected in their spectra. For $\mathrm{C}$ IV, $\mathrm{Mg}$ II, and $\mathrm{H} \alpha$, we fit a power law to the continuum wavelength windows used by $\mathrm{SH} 11$; the flux densities in the BEL wavelength windows (also the same as in SH11) are then normalized by the fitted continuum and integrated to find the EW. For C III], which was not analyzed in SH11, we use continuum windows of 1810-1830 $\AA$ and 1976-1996 ̊, and the BEL window is 1830-1976 $\AA$. The choice of these wavelength windows for C III] is informed by the SDSS composite quasar spectrum of Vanden Berk et al. (2001).

For each BEL, we fit both a broad component and a narrow component, each using a Gaussian with the same line center fixed to their lab vacuum wavelengths. However, the higherionization C IV line is well known to exhibit intrinsic blueshifts (Gaskell 1982; Richards et al. 2002, 2011), and so we allow the line center (assumed to be the same for both broad and narrow components) to be a single free parameter. The FWHM of the narrow component is constrained to be $<1200 \mathrm{~km} \mathrm{~s}^{-1}$, and the amplitudes of all components are constrained to be positive. For $\mathrm{H} \alpha$, we fit additional Gaussians for the narrow [N $\mathrm{II}] \lambda \lambda 6548$, 6584 lines, as well as [S II] $\lambda \lambda 6717,6731$. We visually inspect the fit of each emission line and continuum region to ensure that it was not strongly affected by problematic pixels or absorption features. The statistical uncertainties on all quantities derived from the fitting (notably the EWs, luminosities, and FWHMs) are estimated by simulating the noise in each spectrum, by resampling each pixel from a Gaussian distribution with $\sigma$ set to the uncertainty in the pixel flux density. All pixels in each spectrum are resampled in this fashion and refitted with the above procedure $10^{3}$ times; the uncertainties on the fitted quantities are then based on their $1 \sigma$ range in the simulated spectra. Several studies have indicated a likely dependence of quasar BEL FWHMs on orientation (Wills \& Browne 1986; Jarvis \& McLure 2006; Fine et al. 2011; Runnoe et al. 2013), which may bias our FWHM measurements and black hole mass estimates due to the strong jet-alignment of blazars. However, this bias cannot be significantly larger than the $\lesssim 0.2$ dex dispersion in the log-normal distribution of BEL FWHMs of quasars (Shen et al. 2008), and thus cannot account for the 1-2 dex difference in the Eddington ratio between our transition blazars (see Section 3.1) and that typical of radiatively inefficient accretion.

We identify six blazars for which any BEL in the spectrum crossed the $5 \AA \mathrm{EW}$ threshold in the rest-frame, transitioning between the canonical BLL and FSRQ classifications (or vice versa). The properties of these transition blazars are listed in Table 1 (and discussed in Section 3), and Figures 2-8 show their multi-epoch spectra, the fitting of the continuumnormalized BEL region in each of the epochs, as well as their long-term photometric light curves (discussed in Section 3.4). The observed changes in the EW in the six blazars across the $5 \AA$ threshold vary wildly, up to a factor of $>60$ for SDSS J101603.13+051302.3. Due to the small sample size, we will not attempt to investigate the incidence of these blazar subclass transitions in our current study, and instead focus on understanding the nature of this phenomenon.

Since transition blazars intermittently show small BEL EWs by definition, redshifts can be difficult to obtain from spectra. Indeed, for two of these transition blazars as well as one additional blazar (SDSS J083223.22+491321.0) which did not transition, no previous redshifts have been reported. We report new secure redshifts from the SDSS pipeline using the spectral epochs where the BELs have the largest EWs (are thus most well detected). Although SDSS J083223.22+491321.0 did not transition, we nevertheless show its spectra and BEL fits in Figure 8 due to its newly determined redshift and its special implications for transition blazars (discussed in Section 5). We discuss the properties of each of these individual blazars in more detail in Section 4.

\section{PROPERTIES OF TRANSITION BLAZARS}

\subsection{Emission Line Properties}

The homogeneous nature of SDSS spectra allows us not only to identify a sample of transition blazars with well-determined redshifts, but also to characterize robustly their emission line properties. Using the BEL fits described in Section 2.1, we calculate their intrinsic line luminosities over their corresponding BEL wavelength windows; these line luminosities are also listed in Table 1. Figure 9 (left panel) shows the BEL EW versus the fitted continuum luminosities in the BEL wavelength windows, for all epochs of the BELs of each transition blazar in temporal order. A clear anti-correlation is present across different BELs and in each of the different blazars for nearly all epochs. This anti-correlation strongly suggests that the EW variability causing the transition blazar phenomenon in our sample is primarily due to swamping of the emission lines by the jet continuum emission, which is highly variable due to Doppler boosting. However, the emission lines themselves can also have intrinsic variability in their luminosities, which could contribute to their observed EW changes. Figure 9 (right panel) shows the line luminosity versus the continuum luminosity of the same lines as the left panel. The lines are variable up to $\gtrsim 0.5$ dex in luminosity; in some instances (e.g., SDSS J125032.57+021632.1), the BEL luminosities appear to show an additional anti-correlation with the continuum luminosity (i.e., the lines become more luminous as the continuum luminosity decreases), further increasing the EW variability. Variations in luminosity of BELs of $0.6 \mathrm{dex}$ have also been previously observed in the namesake BLL object (Corbett et al. 1996), which is a known transition blazar.

We use the line luminosities to estimate the bolometric Eddington ratio $\left(L_{\mathrm{bol}} / L_{\mathrm{Edd}}\right)$ as well as the Eddington ratio of the broad line emission $\left(L_{\mathrm{BLR}} / L_{\mathrm{Edd}}\right)$. These two quantities which 
Table 1

SDSS Spectral Properties of All Broad Emission Lines Present in Each of the Transition Blazars in Our Sample

\begin{tabular}{|c|c|c|c|c|c|c|c|c|c|}
\hline $\begin{array}{l}\text { SDSS } \\
\text { Object }\end{array}$ & $z$ & $\begin{array}{c}\text { Emission } \\
\text { Line }\end{array}$ & MJD & $\begin{array}{l}\text { EW } \\
(\AA)\end{array}$ & $\begin{array}{c}\log _{10} L_{\text {line }} \\
\left(\mathrm{erg} \mathrm{s}^{-1}\right)\end{array}$ & $\begin{array}{c}\text { FWHM } \\
\left(\mathrm{km} \mathrm{s}^{-1}\right)\end{array}$ & $\begin{array}{c}\log _{10} M_{\mathrm{BH}} \\
\left(M_{\odot}\right)\end{array}$ & $\begin{array}{c}\log _{10}\left(L_{\text {bol }}\right. \\
\left./ L_{\text {Edd }}\right)\end{array}$ & $\begin{array}{c}\log _{10}\left(L_{\mathrm{BLR}}\right. \\
\left./ L_{\mathrm{Edd}}\right)\end{array}$ \\
\hline \multirow[t]{3}{*}{$\mathrm{J} 083223.22+491321.0^{\mathrm{a}}$} & $0.548^{\mathrm{b}}$ & Mg II & 51873 & $0.9 \pm 0.7$ & $41.52 \pm 0.02$ & & & & \\
\hline & & & 55277 & $1.3 \pm 0.3$ & $41.47 \pm 0.01$ & & & & \\
\hline & & & 55290 & $2.3 \pm 0.5$ & $41.59 \pm 0.01$ & $2126 \pm 332$ & $6.84 \pm 0.15$ & $-0.76 \pm 0.23$ & $-2.14 \pm 0.15$ \\
\hline \multirow[t]{3}{*}{ J083353.88+422401.8 } & $0.249^{c}$ & $\mathrm{H} \alpha$ & 52266 & $5.1 \pm 0.6$ & $41.63 \pm 0.05$ & $809 \pm 228$ & $6.33 \pm 0.26$ & $0.18 \pm 0.48$ & $-1.94 \pm 0.26$ \\
\hline & & & 54524 & $4.8 \pm 0.8$ & $41.71 \pm 0.07$ & & & & \\
\hline & & & 55924 & $2.1 \pm 0.3$ & $41.68 \pm 0.06$ & & & & \\
\hline \multirow[t]{15}{*}{$\mathrm{J} 101603.13+051302.3$} & $1.714^{\mathrm{c}}$ & CiV & 52347 & $1.8 \pm 0.2$ & $43.12 \pm 0.04$ & & & & \\
\hline & & & 52355 & $14.8 \pm 0.4$ & $43.91 \pm 0.01$ & & & & \\
\hline & & & 52356 & $43.4 \pm 1.6$ & $43.94 \pm 0.01$ & & & & \\
\hline & & & 52366 & $19.56 \pm 5.3$ & $43.70 \pm 0.09$ & & & & \\
\hline & & & 55653 & $116.8 \pm 4.3$ & $43.76 \pm 0.01$ & $3267 \pm 50$ & $8.44 \pm 0.22$ & $-0.54 \pm 0.61$ & $-1.83 \pm 0.22$ \\
\hline & & C III] & 52347 & $1.0 \pm 0.1$ & $42.75 \pm 0.05$ & & & & \\
\hline & & & 52355 & $4.0 \pm 0.4$ & $42.26 \pm 0.04$ & & & & \\
\hline & & & 52356 & $3.3 \pm 0.4$ & $42.83 \pm 0.04$ & & & & \\
\hline & & & 52366 & $10.8 \pm 0.8$ & $43.33 \pm 0.03$ & & & & \\
\hline & & & 55653 & $55.2 \pm 3.0$ & $43.32 \pm 0.02$ & & & & \\
\hline & & Mg II & 52347 & $1.7 \pm 0.1$ & $42.85 \pm 0.03$ & & & & \\
\hline & & & 52355 & $5.3 \pm 0.3$ & $43.30 \pm 0.02$ & & & & \\
\hline & & & 52356 & $15.8 \pm 1.7$ & $43.39 \pm 0.05$ & & & & \\
\hline & & & 52366 & $8.6 \pm 0.6$ & $43.16 \pm 0.03$ & & & & \\
\hline & & & 55653 & $72.9 \pm 4.6$ & $43.37 \pm 0.03$ & $2739 \pm 224$ & $8.18 \pm 0.10$ & $-0.30 \pm 0.20$ & $-1.70 \pm 0.10$ \\
\hline \multirow[t]{2}{*}{$\mathrm{J} 125032.57+021632.1$} & $0.959^{\mathrm{b}}$ & Mg II & 52024 & $3.6 \pm 0.4$ & $42.29 \pm 0.05$ & & & & \\
\hline & & & 55631 & $8.2 \pm 0.2$ & $42.54 \pm 0.01$ & $4360 \pm 213$ & $8.06 \pm 0.08$ & $-1.01 \pm 0.19$ & $-2.41 \pm 0.08$ \\
\hline \multirow[t]{2}{*}{$\mathrm{J} 130823.70+354637.0$} & $1.055^{\mathrm{c}}$ & Mg II & 55335 & $8.5 \pm 1.2$ & $42.46 \pm 0.06$ & $2250 \pm 718$ & $7.43 \pm 0.29$ & $-0.46 \pm 0.34$ & $-1.86 \pm 0.30$ \\
\hline & & & 55597 & $4.9 \pm 0.7$ & $42.74 \pm 0.06$ & & & & \\
\hline \multirow[t]{2}{*}{$\mathrm{J} 143758.67+300207.1$} & $1.230^{\mathrm{b}}$ & Mg II & 53757 & $0.4 \pm 0.2$ & $42.14 \pm 0.02$ & & & & \\
\hline & & & 55277 & $25.6 \pm 0.5$ & $42.76 \pm 0.01$ & $3829 \pm 281$ & $8.08 \pm 0.09$ & $-0.81 \pm 0.19$ & $-2.21 \pm 0.09$ \\
\hline \multirow[t]{2}{*}{$\mathrm{J} 220643.28-003102.5$} & $1.051^{\mathrm{d}}$ & Mg II & 52937 & $9.9 \pm 1.5$ & $42.81 \pm 0.06$ & $4189 \pm 1683$ & $8.19 \pm 0.36$ & $-0.87 \pm 0.40$ & $-2.27 \pm 0.36$ \\
\hline & & & 55481 & $4.9 \pm 0.8$ & $42.61 \pm 0.07$ & & & & \\
\hline
\end{tabular}

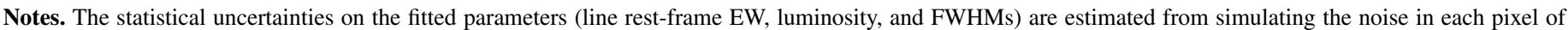

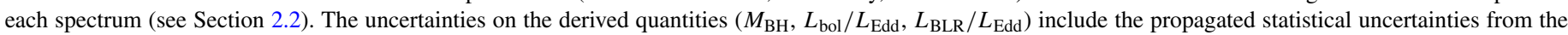
fitting, as well as all statistical and systematic uncertainties in the scaling relations used in Section 3.1.

a This blazar did not transition, but is likely to be a transition blazar (see Section 4).

b New redshift.

${ }^{c}$ Redshift from Massaro et al. (2009).

${ }^{\mathrm{d}}$ Redshift from Shaw et al. (2013b).

probe the accretion state are often used to investigate blazar subclasses in large samples (e.g., Ghisellini et al. 2011; Shaw et al. 2012), allowing us to place the accretion state of our transition blazars in a broader context. We calculate $L_{\text {bol }} / L_{\mathrm{Edd}}$ of the transition blazars in our sample following the method of Shaw et al. (2012), largely based on the results of SH11. We first estimate the unbeamed thermal continuum luminosity at $3000 \AA$ using its correlation with the $\mathrm{Mg}$ II line luminosity determined by SH11 from a large sample of quasars,

$$
\log \left(\frac{L_{\text {cont }}}{\operatorname{erg~s}^{-1}}\right)=a \cdot \log \left(\frac{L_{\text {line }}}{\operatorname{erg~s}^{-1}}\right)+b
$$

where $a=1.016 \pm 0.003$ and $b=1.22 \pm 0.11$ for the $\mathrm{Mg}$ II BEL. We use this correlation to estimate the continuum luminosity rather than a direct measurement because the continuum emission in blazars is often dominated by non-thermal jet emission. The bolometric luminosity is estimated by multiplying the continuum luminosity at $3000 \AA$ from Equation (1) by the bolometric correction of $L_{\mathrm{bol}} / L_{3000}=5.15$ from Richards et al. (2006). Using the luminosity and the fitted FWHMs of the Mg II lines, we estimate black hole masses $M_{\mathrm{BH}}$ using the relation

$$
\log \left(\frac{M_{\mathrm{BH}}}{M_{\odot}}\right)=c+d \cdot \log \left(\frac{L_{\text {line }}}{10^{44} \mathrm{erg} \mathrm{s}^{-1}}\right)+2 \log \left(\frac{\mathrm{FWHM}}{\mathrm{km} \mathrm{s}^{-1}}\right),
$$

where $c=1.70 \pm 0.07$ and $d=0.63 \pm 0.00$, also determined by SH11.

For the low- $z$ blazar SDSS J083353.88+422401.8, Mg II is not in the observed spectral wavelength range, and so the Eddington ratio must be calculated using other BELs. $\mathrm{H} \alpha$ is the only measurable BEL in this blazar, as $\mathrm{H} \beta$ is too weak to be discernible from the continuum, even in the low continuum luminosity epochs. We thus estimate $M_{\mathrm{BH}}$ using its relation with the $\mathrm{H} \alpha$ line luminosity and FWHM, determined by SH11 (their Equation (10)) and based on Greene \& Ho (2005). Because SH11 did not measure correlations between continuum and line luminosities for $\mathrm{H} \alpha$, we estimate the continuum luminosity $L_{5100}$ by first estimating the FWHM of $\mathrm{H} \beta$ (not observable) using its correlation with the $\mathrm{H} \alpha$ FWHM determined in SH11. We can then estimate $L_{5100}$ from its relation with the $M_{\mathrm{BH}}$ and the $\mathrm{H} \beta$ FWHM, using Equation (5) from Shaw et al. (2012) with their constants $a=0.672$ and $b=0.61$, determined by 

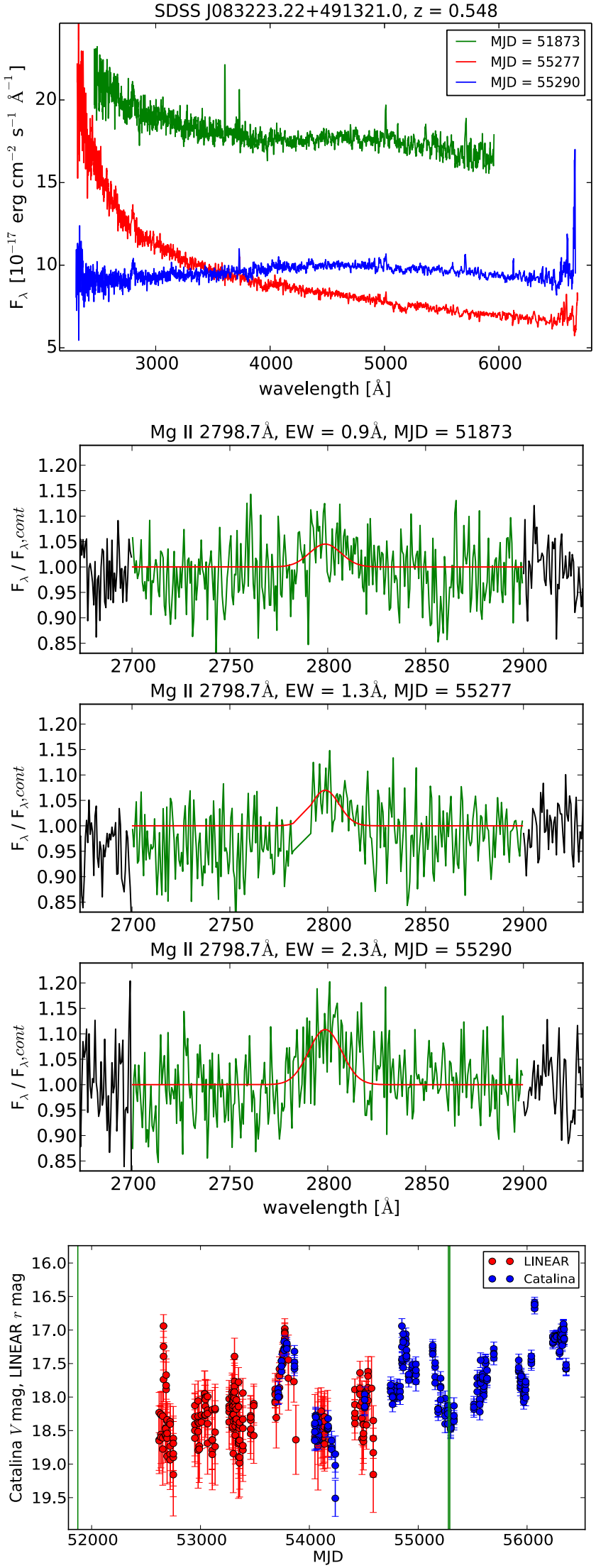

Figure 2. SDSS J083223.22+491321.0: multi-epoch observed-frame SDSS spectra, rest-frame broad emission line fitting, and photometric light curve. The dates of the spectroscopic epochs are indicated by the green lines in the light curve.

(A color version of this figure is available in the online journal.)
McLure \& Dunlop (2004). Finally, the bolometric luminosity is calculated using $L_{\text {bol }} / L_{5100}=9.26$ (Richards et al. 2006).

We estimate the $M_{\mathrm{BH}}$ and $L_{\mathrm{bol}} / L_{\mathrm{Edd}}$ of each blazar using the spectral epochs for which the BELs have the largest EWs; these values are also listed in Table 1. All uncertainties on these derived quantities include the propagated statistical uncertainties from the spectral line fitting, as well as the reported statistical and systematic uncertainties of the constants in each of the scaling relations. We are also able to estimate $L_{\text {bol }}$ and $M_{\mathrm{BH}}$ for SDSS J101603.13+051302.3 using C IV independently in addition to $\mathrm{Mg}$ II; this calculation is performed using $a=0.863 \pm 0.009$ and $b=7.66 \pm 0.41$ in Equation (1) (SH11) where the line luminosity is for C IV and the continuum luminosity is for $1350 \AA$, and using a bolometric correction of $L_{\mathrm{bol}} / L_{1350}=3.81$ (Richards et al. 2006). The $M_{\mathrm{BH}}$ based on $\mathrm{C}$ IV is then calculated by setting $c=1.52 \pm 0.22$ and $d=0.46 \pm 0.01$ in Equation (2) (SH11). The $M_{\mathrm{BH}}$ and $L_{\mathrm{bol}} /$ $L_{\text {Edd }}$ calculated independently from $\mathrm{C}$ IV and $\mathrm{Mg}$ II in this object are consistent to within the uncertainty.

We also estimate the total luminosity of the broad line emission to estimate the Eddington ratio of the BELs ( $L_{\mathrm{BLR}} /$ $L_{\text {Edd }}$ ). This calculation is performed in a manner similar to the method of Celotti et al. (1997), who found that the ratio of the total luminosity of all BELs to the line luminosities $\left(L_{\mathrm{BLR}} /\right.$ $\left.L_{\text {line }}\right)$ for $\mathrm{H} \alpha, \mathrm{Mg}$ II, and $\mathrm{CIV}$ are 555.76/77, 555.76/34, and $555.76 / 63$, respectively, based primarily on BEL flux ratios in the quasar composite spectrum of Francis et al. (1991). Using these factors, we calculate $L_{\mathrm{BLR}}$ for each of our transition blazars at the spectral epoch with largest EW, similar to that done for $L_{\text {bol }} / L_{\text {Edd }}$ above. For SDSS J101603.13+051302.3, the $L_{\text {BLR }}$ independently based on $\mathrm{C}$ IV is also highly consistent with $\mathrm{Mg}$ II. Although a single measurement of $L_{\mathrm{bol}} / L_{\mathrm{Edd}}$ is most helpful for classification in cases where multiple BELs are available (e.g., by averaging estimates derived from multiple lines), we opt to use the estimate from $\mathrm{Mg}$ II as the fiducial $L_{\text {bol }} / L_{\text {Edd }}$ for J101603.13+051302.3 due to its smaller scatter in the $M_{\mathrm{BH}}$ scaling relations we use in comparison to that of $\mathrm{C} \mathrm{IV}$, which instead serves as a helpful consistency check. Figure 10 presents the bolometric Eddington ratio of the transition blazars against the Eddington ratio of the BELs for the transition blazars in our sample, as well as for the blazar which did not transition. The Eddington ratios of these blazars are similar to the Eddington ratios of typical FSRQs (and much larger than those from radiatively inefficient accretion flows in BLLs). We discuss our interpretation of these results in Section 5.

\subsection{Spectral Energy Distribution Properties}

Blazars are well known to emit strongly at a wide range of wavelengths, and their SEDs are dominated by synchrotron emission at radio to soft X-ray wavelengths and inverseCompton emission at hard X-ray to $\gamma$-ray wavelengths. The peaks of the synchrotron and inverse-Compton emission tend to occur at lower frequencies for FSRQs than for BLLs in general, although BLLs have often been further subdivided into low-, intermediate-, and high-synchrotron peaked BLLs, leading to the idea of a possible continuous "blazar sequence" of SEDs (Fossati et al. 1998; Ghisellini \& Tavecchio 2008). We compile SEDs for the transition blazars in our sample using the ASI Science Data Center Virtual Observatory SED builder ${ }^{8}$ (Stratta et al. 2011), conveniently linked to all blazars in the ROMABZCAT catalog. The archival multi-wavelength data available

\footnotetext{
8 http://tools.asdc.asi.it/SED/
} 
Table 2

$\gamma$-Ray and Long-term Photometric Variability Properties of the Transition Blazars in Our Sample

\begin{tabular}{|c|c|c|c|c|c|c|}
\hline $\begin{array}{l}\text { SDSS } \\
\text { Object }\end{array}$ & $\begin{array}{l}\log _{10} L_{\gamma}{ }^{a} \\
\left(\mathrm{erg} \mathrm{s}^{-1}\right)\end{array}$ & $\alpha_{\gamma}{ }^{b}$ & $\begin{array}{c}\log _{10} v_{\text {peak }}^{\mathrm{c}} \\
(\mathrm{Hz})\end{array}$ & $\begin{array}{c}\log _{10} L_{\text {peak }}^{\mathrm{d}} \\
\left(\mathrm{erg} \mathrm{s}^{-1}\right)\end{array}$ & $\begin{array}{c}\log _{10} \mathrm{SF}_{\infty} \mathrm{e} \\
(\mathrm{mag})\end{array}$ & $\begin{array}{l}\log _{10} \tau^{\mathrm{f}} \\
\text { (days) }\end{array}$ \\
\hline J083223.22+491321.0 & & & 13.12 & 45.51 & -0.14 & 1.33 \\
\hline J083353.88+422401.8 & 45.22 & 2.33 & 14.11 & 44.80 & -0.21 & 1.38 \\
\hline $\mathrm{J} 101603.13+051302.3$ & 47.99 & 2.08 & 13.27 & 45.94 & 0.31 & 0.79 \\
\hline $\mathrm{J} 125032.57+021632.1$ & & & 13.74 & 45.49 & 0.05 & 1.76 \\
\hline $\mathrm{J} 130823.70+354637.0$ & 46.89 & 2.28 & 12.88 & 45.89 & -0.09 & 0.81 \\
\hline J143758.67+300207.1 & & & & & 0.13 & 0.93 \\
\hline $\mathrm{J} 220643.28-003102.5$ & 46.61 & 2.16 & 13.04 & 46.02 & -0.06 & 0.78 \\
\hline
\end{tabular}

Notes.

a $0.1-100 \mathrm{GeV}$ luminosity from Nolan et al. (2012)

b $0.1-100 \mathrm{GeV}$ photon number power-law index from Nolan et al. (2012)

${ }^{c}$ Estimated frequency of the synchrotron peak in the SED.

${ }^{\mathrm{d}}$ Estimated luminosity of the synchrotron peak in the SED.

e Optical photometric variability amplitude on long timescales.

${ }^{\mathrm{f}}$ Characteristic timescale of optical photometric variability in the rest-frame.

are most complete at radio to optical wavelengths, and visual inspection of the SEDs of our sample of transition blazars shows that the synchrotron peak is well determined for all but one (SDSS J143758.67+300207.1), which has few data points in its SED.

We estimate the frequency and luminosity of the synchrotron peak of the transition blazars (except for SDSS J143758.67+300207.1) by fitting a third-order polynomial to the synchrotron emission in the SEDs. In the fitting, we have purposely excluded data points at frequencies $>10^{17} \mathrm{~Hz}$ to avoid inverse-Compton emission. The estimated synchrotron peak frequencies and luminosities are listed in Table 2. These order-of-magnitude estimates are approximate since we are not fitting a physically motivated model to the SED and the archival SED data points are not contemporaneous. However, the SED peaks are reasonably well determined, and sufficient for classification of SED type. All the measured peak frequencies of these transition blazars are similar to the $10^{13-14} \mathrm{~Hz}$ range of low-synchrotron peaked blazars, and their luminosities are also consistent with the $>10^{43} \mathrm{erg} \mathrm{s}^{-1}$ luminosities of blazars in this SED class. This result is in strong contrast to the $\sim 10^{14-16} \mathrm{~Hz}$ and $\sim 10^{16-18} \mathrm{~Hz}$ synchrotron peak frequencies of intermediateand high-synchrotron peaked blazars, respectively (Abdo et al. 2010a). Thus, the SED properties of these transition blazars (as well as those of SDSS J083223.22+491321.0) are consistent with FSRQs and low-synchrotron peaked BLLs.

\section{3. $\gamma$-Ray Properties}

The Large Area Telescope (Atwood et al. 2009) on board Fermi has surveyed the $\gamma$-ray sky to unprecedented depth, thus far detecting $1873 \gamma$-ray sources in the two year 2FGL catalog (Nolan et al. 2012), including 1121 AGNs in the second Fermi AGN catalog (Ackermann et al. 2011). The overwhelming majority of Fermi-detected AGNs are blazars, approximately evenly divided between FSRQs and BLLs. Analysis of these Fermi blazars has revealed that BLLs tend to have $\gamma$-ray luminosities $L_{\gamma} \lesssim 10^{46} \mathrm{erg} \mathrm{s}^{-1}$ and $\gamma$-ray spectral indices $\alpha_{\gamma} \lesssim$ 2.2 in the $0.1-100 \mathrm{GeV}$ band, while FSRQs exceed these values. However, there is significant overlap in these $\gamma$-ray properties between the two subclasses (Ackermann et al. 2011), likely due to some combination of large measurement uncertainties and misclassification, in addition to astrophysical origins.
We match our transition blazars to the Fermi 2FGL catalog to investigate their $\gamma$-ray properties. Four of the blazars in our sample are matched to 2FGL sources that have association probabilities $>95 \%$ (i.e., the blazar is highly likely to be the optical counterpart of the $\gamma$-ray source); their $\gamma$-ray luminosities and spectral indices are also listed in Table 2 . None of the four transition blazars in our sample detected by Fermi can be clearly classified based on their $\gamma$-ray properties, and all lie in the area occupied by FSRQs and low-to-intermediate synchrotron peaked BLLs. Ghisellini et al. (2011) argued that for such objects, a better classifier to use is the Eddington ratio based on the BEL $\left(L_{\mathrm{BLR}} / L_{\mathrm{Edd}}\right)$, with a dividing value of $\sim 5 \times 10^{-4}$. We note that although the synchrotron peak frequencies and luminosities of these transition blazars (where determined) are similar, not all are detected in $\gamma$-rays.

\subsection{Optical Variability Properties}

Blazars are well known to be among the most variable extragalactic objects detected in large-scale optical time-domain surveys (Bauer et al. 2009). An investigation of long-term optical light curves recalibrated by Sesar et al. (2011) from the Lincoln Near-Earth Asteroid Research (LINEAR; Stokes et al. 2000) survey by Ruan et al. (2012) revealed that the jet synchrotrondominated continuum emission in blazars is systematically more variable than the thermal disk-dominated continuum in typical Type 1 quasars. In particular, Ruan et al. (2012) found that blazars have typical optical variability amplitudes of $\sim 0.5 \mathrm{mag}$ in the $r$-band on rest-frame timescales greater than $\sim 100$ days; below this characteristic timescale, the optical variability is selfcorrelated and smaller in amplitude. The strong variability amplitudes and short variability timescales observed in comparison to typical quasars was interpreted by Ruan et al. (2012) as due to the effects of Doppler boosting in the relativistic jet of blazars.

We compile publicly available optical light curves of the transition blazars in our sample from the LINEAR and Catalina Sky Survey/Catalina Real-Time Transient Survey (Drake et al. 2009) databases to investigate their optical variability properties. The optical light curves constructed using these two surveys are also shown in Figures 2-8; they are well sampled, with 98-978 epochs (median of 444 epochs) spread over $\sim 11 \mathrm{yr}$ in the observed frame. All transition blazars in our sample display strong optical variability with maximum amplitude in the range of $\sim 1.5-6$ mag in the data. 
To compare the optical variability properties of these transition blazars to the large sample of FSRQs and BLLs from Ruan et al. (2012), we fit each of these light curves to a firstorder continuous autoregressive model (i.e., a damped random walk) using the method of Kozłowski et al. (2010), and discussed in detail in MacLeod et al. (2010). Figure 11 compares the rest-frame characteristic timescale of variability $\tau$, and the variability amplitude on long timescales (when the variability is uncorrelated and strongest) $\mathrm{SF}_{\infty}$, of these seven blazars to the large sample of 60 bright blazars from Ruan et al. (2012). The seven blazars in our sample have larger variability amplitudes and shorter timescales of variability than typical blazars. A twosample Kolmogorov-Smirnov test between the distributions of $\tau$ and $\mathrm{SF}_{\infty}$ in Figure 11 for our transition blazars and the blazar sample of Ruan et al. (2012) results in a $p$ value of $3.1 \times 10^{-3}$ for $\tau$ and $5.6 \times 10^{-4}$ for $\mathrm{SF}_{\infty}$.

Although the light curves we construct use data from both LINEAR and the Catalina surveys, the filters and calibrations are not exact between these two surveys; this issue will lead to a systematic offset between their measured magnitudes. However, both surveys used white-light filters and the systematic offset in magnitude is small ( $<0.1 \mathrm{mag}$ ), especially in comparison to the several-magnitude variability observed in our transition blazar light curves. Thus, any differences in calibration between the two surveys are dwarfed by the intrinsic variability of transition blazars and do not significantly affect our analysis.

\section{NOTES ON INDIVIDUAL OBJECTS}

SDSS J083223.22+491321.0. The Mg II line EW of this blazar is $<5 \AA$ in all three available epochs of spectra (Figure 8), and thus this blazar appears to be a canonical BLL and was included in our analysis for completeness due to its newly determined redshift of $z=0.548$. However, despite its small $\mathrm{Mg}$ II EW and under-luminous $\mathrm{Mg}$ II emission relative to the FSRQs in Shaw et al. (2012), its high bolometric Eddington ratio instead strongly suggests that it is in fact an FSRQ and would transition with further spectroscopic monitoring. This blazar is not Fermi-detected.

SDSS J083353.88+422401.8. The $\mathrm{H} \alpha$ line is visible in this low-redshift blazar in all three available epochs of spectra (Figure 2), although its EW is small in even the largest EW epoch. Giommi et al. (2012) suggested that blazars with BELs that transition in their optical spectra likely have highly luminous $\mathrm{H} \alpha$ emission in the observed-frame infrared with EWs that remain $>5 \AA$. However, this blazar is an example in which even the $\mathrm{H} \alpha$ emission transitions. The $M_{\mathrm{BH}}$ and $\mathrm{H} \alpha$ luminosity we calculate for this blazar are smaller than typical of FSRQs in the sample of Shaw et al. (2012), although this may be affected by our indirect method of estimating the continuum luminosity through correlations between the $\mathrm{H} \alpha$ and the unobserved $\mathrm{H} \beta$ BELs (see Section 3.1). To verify our $M_{\mathrm{BH}}$ estimate, we also fit the $\mathrm{H} \alpha \mathrm{FWHM}$ in the second-largest EW spectral epoch $(\mathrm{MJD}=$ 54524) for this blazar and compare to the largest EW epoch. The FWHMs of both epochs are highly consistent, and thus our relatively small $M_{\mathrm{BH}}$ estimate appears to be reliable. This blazar is Fermi-detected, and its optical light curve is exceptionally well sampled, showing variability up to $\sim 2 \mathrm{mag}$.

SDSS J101603.13+051302.3. The C IV, C III], and MgII BELs all show strong variability in EW (up to a factor of $>60$ ) over five available epochs of SDSS spectra (Figure 3). The decrease in continuum luminosity in these spectra causes the BELs to transition nearly in unison. This strong EW variability is not particularly surprising, as the long-term continuum
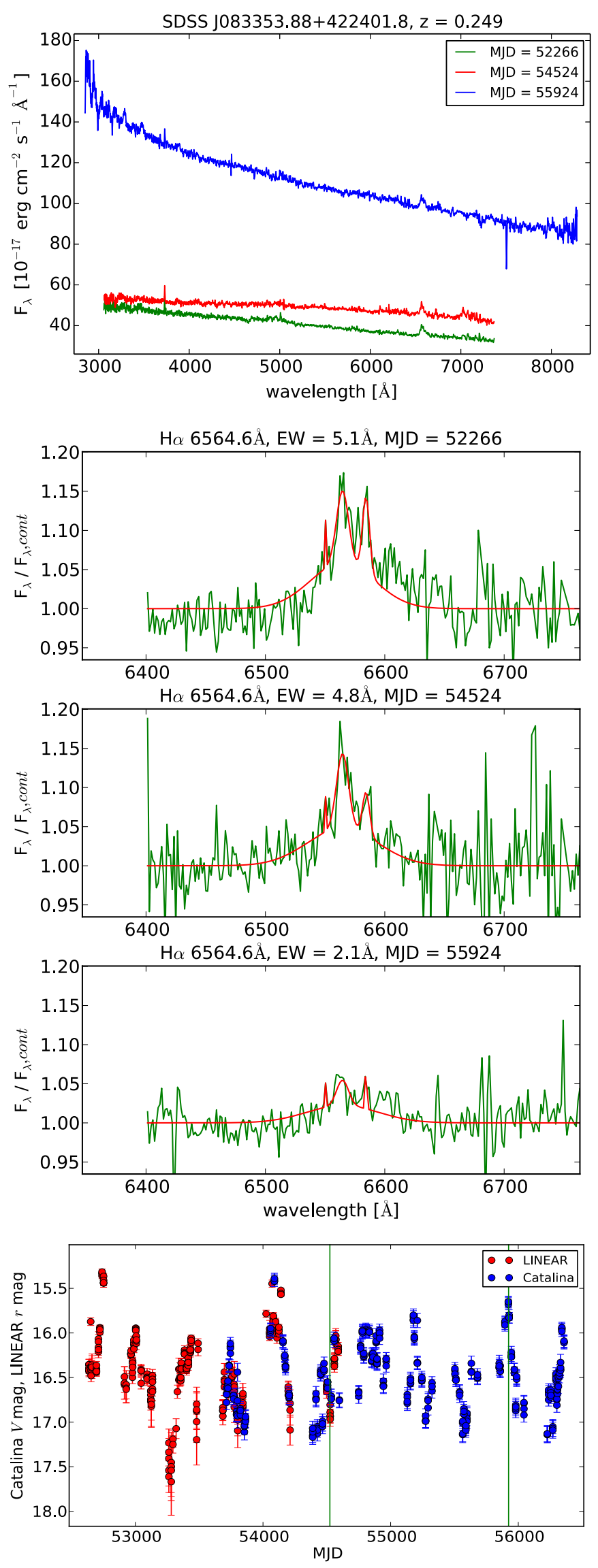

Figure 3. SDSS J083353.88+42241.8, similar to Figure 2. (A color version of this figure is available in the online journal.) 

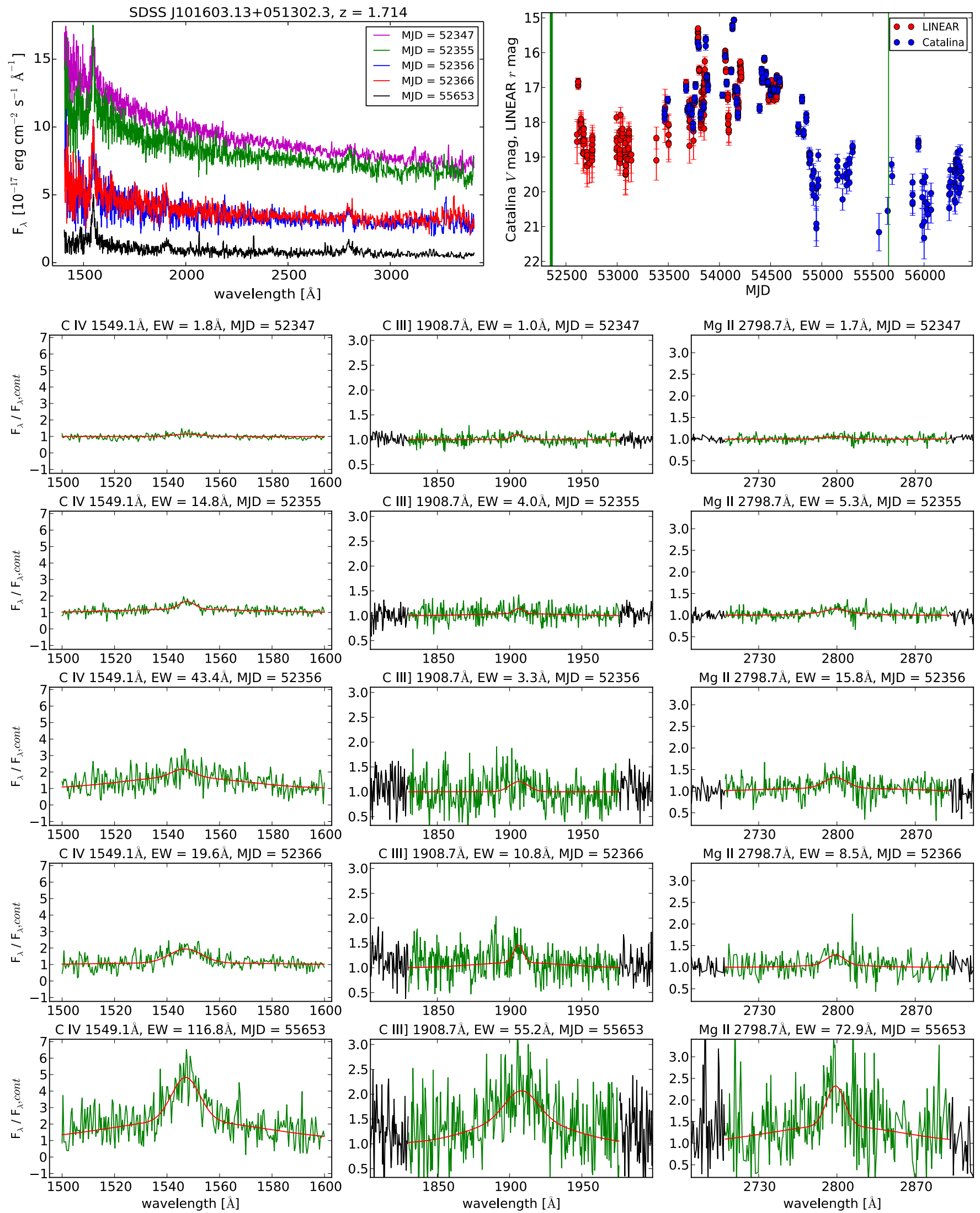

Figure 4. SDSS J10163.13+05132.3, similar to Figure 2.

(A color version of this figure is available in the online journal.) 

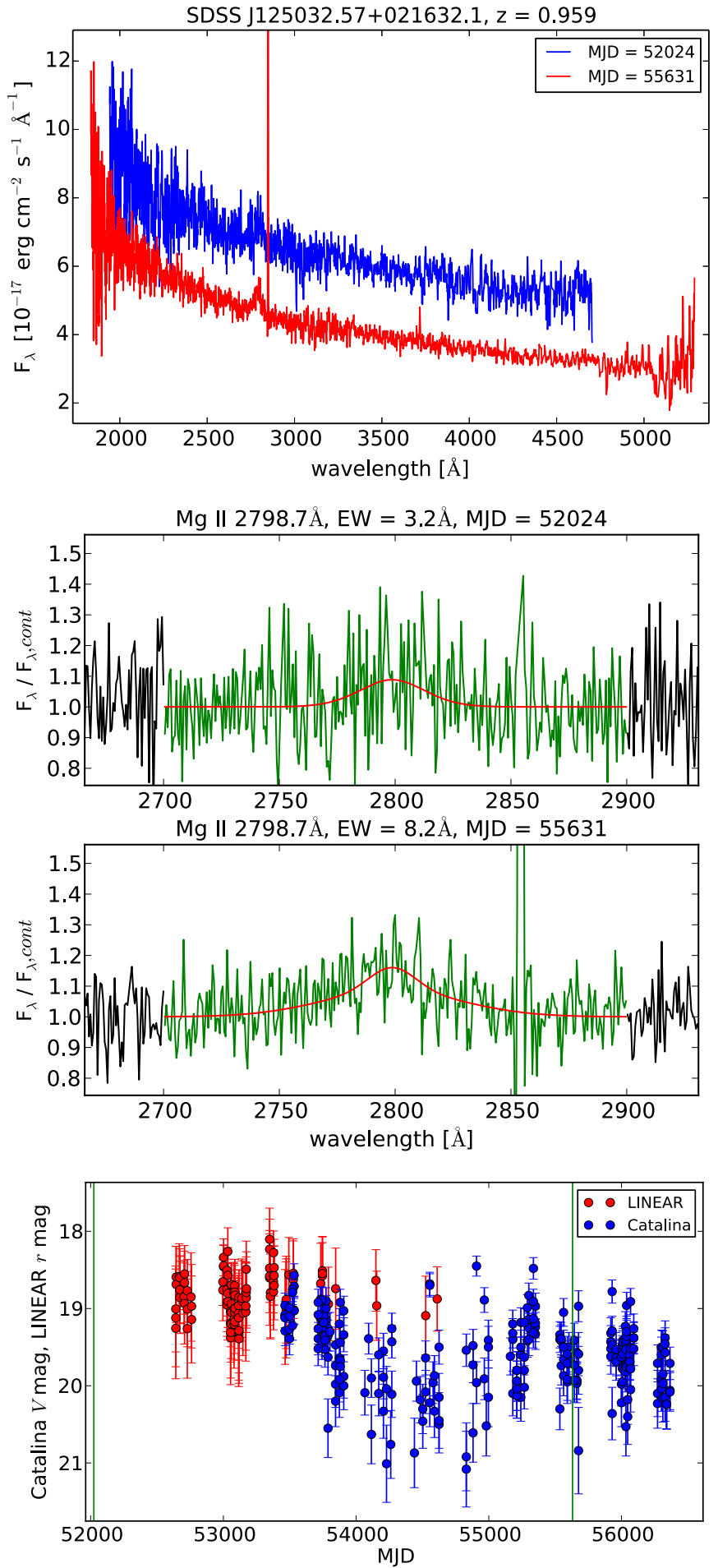

Figure 5. SDSS J125032.57+021632.1, similar to Figure 2. (A color version of this figure is available in the online journal.)

variability probed by the broadband optical light curve varies up to $\gtrsim 5$ mag. This blazar is a bright Fermi source, and its bolometric Eddington ratio determined from $\mathrm{Mg}$ II (more robust than that from C IV) shows that it is accreting even more rapidly than typical FSRQs.

SDSS J125032.57+021632.1. Although this blazar did not previously have a redshift in the ROMA-BZCAT catalog, the $\mathrm{Mg}$ II line is well detected in both epochs of spectra (Figure 4), and we determine a new robust redshift of $z=0.959$. This
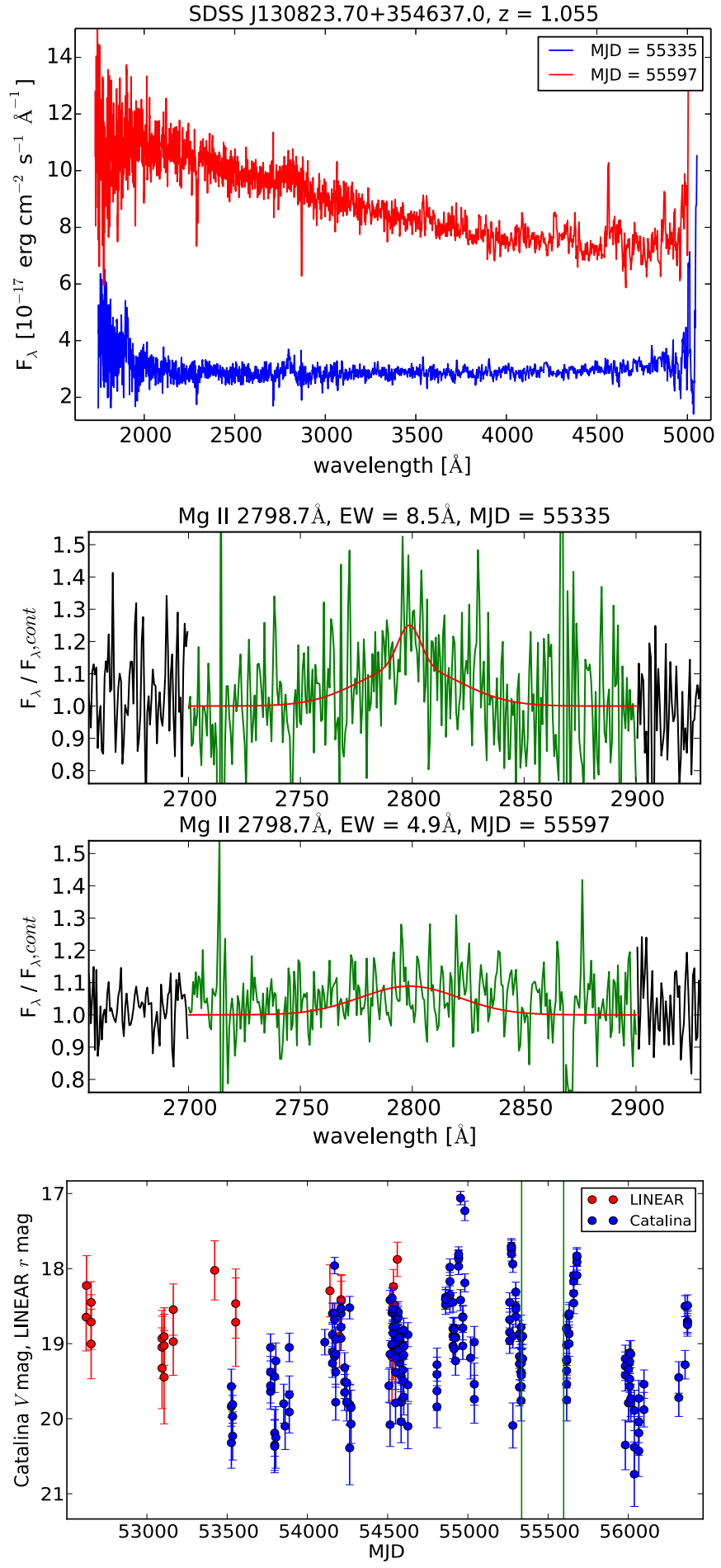

Figure 6. SDSS J130823.70+354637.0, similar to Figure 2.

(A color version of this figure is available in the online journal.)

blazar is not Fermi detected and has an Eddington ratio of $\sim 0.1$, more similar to typical quasars than FSRQs.

SDSS J130823.70+354637.0. The two epochs of spectra for this blazar are observed during dramatic dimming and flaring events in its optical light curve (Figure 5). These dramatic flares of $\sim 2$ mag appear to be a frequent occurrence for this blazar, on timescales as short as days to weeks. The $\mathrm{Mg}$ II line is well detected in both epochs, and this blazar is Fermi-detected. 

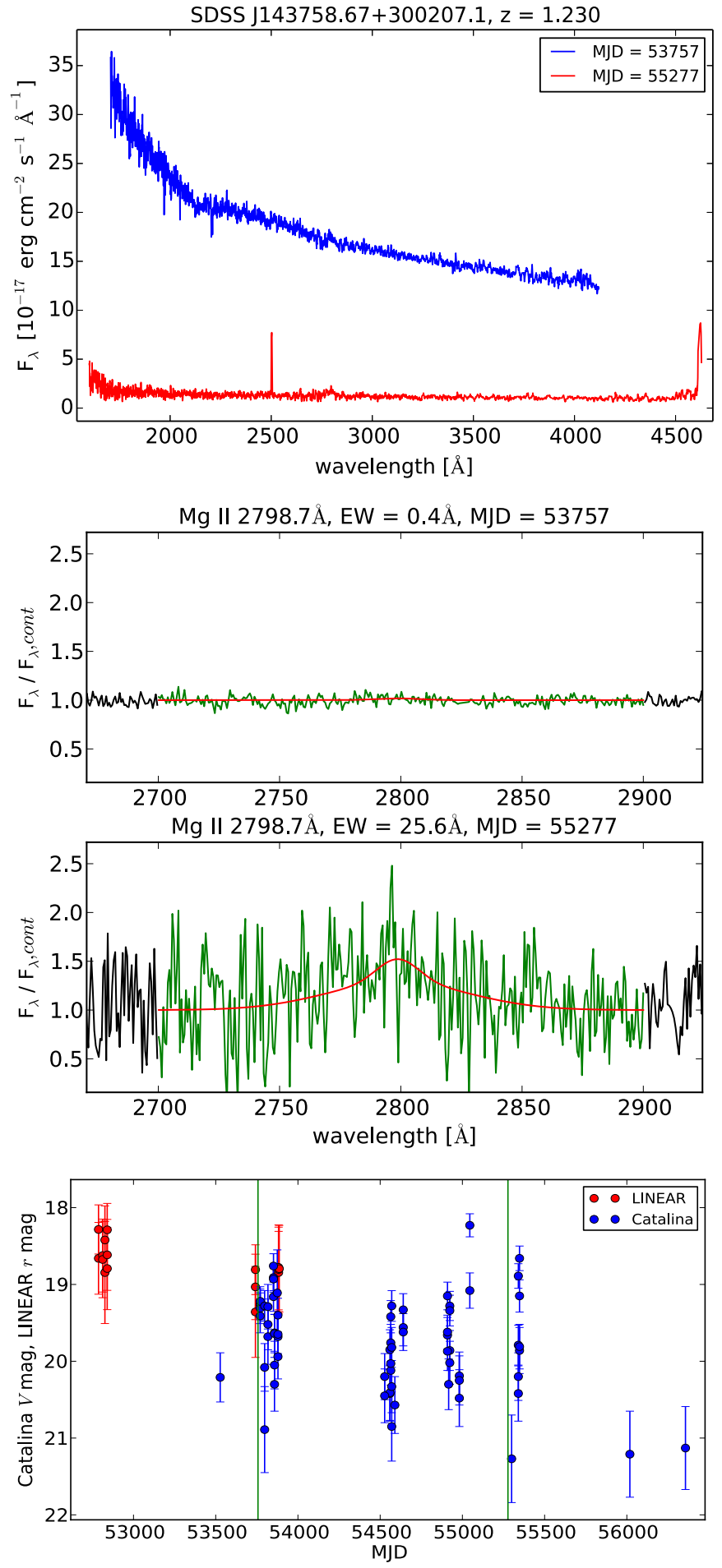

Figure 7. SDSS J143758.67+30027.1, similar to Figure 2. (A color version of this figure is available in the online journal.)

SDSS J143758.67+300207.1. The Mg II line in this blazar is weak in both epochs of spectra (Figure 6), but the strong continuum variability causes its EW to increase by a factor of $>60$. The redshift for this blazar $(z=1.230)$ is new, and this blazar is not Fermi detected. Although the optical light curve is sparsely sampled, large variations of up to $\sim 3$ mag are evident.

SDSS J220643.28-003102.5. This Fermi-detected blazar's $\mathrm{Mg}$ II line is much more clearly detected in the fainter-continuum epoch (Figure 7). The optical light curve shows strong variability with large $(\sim 2 \mathrm{mag})$ flares on timescales of days to weeks. Its
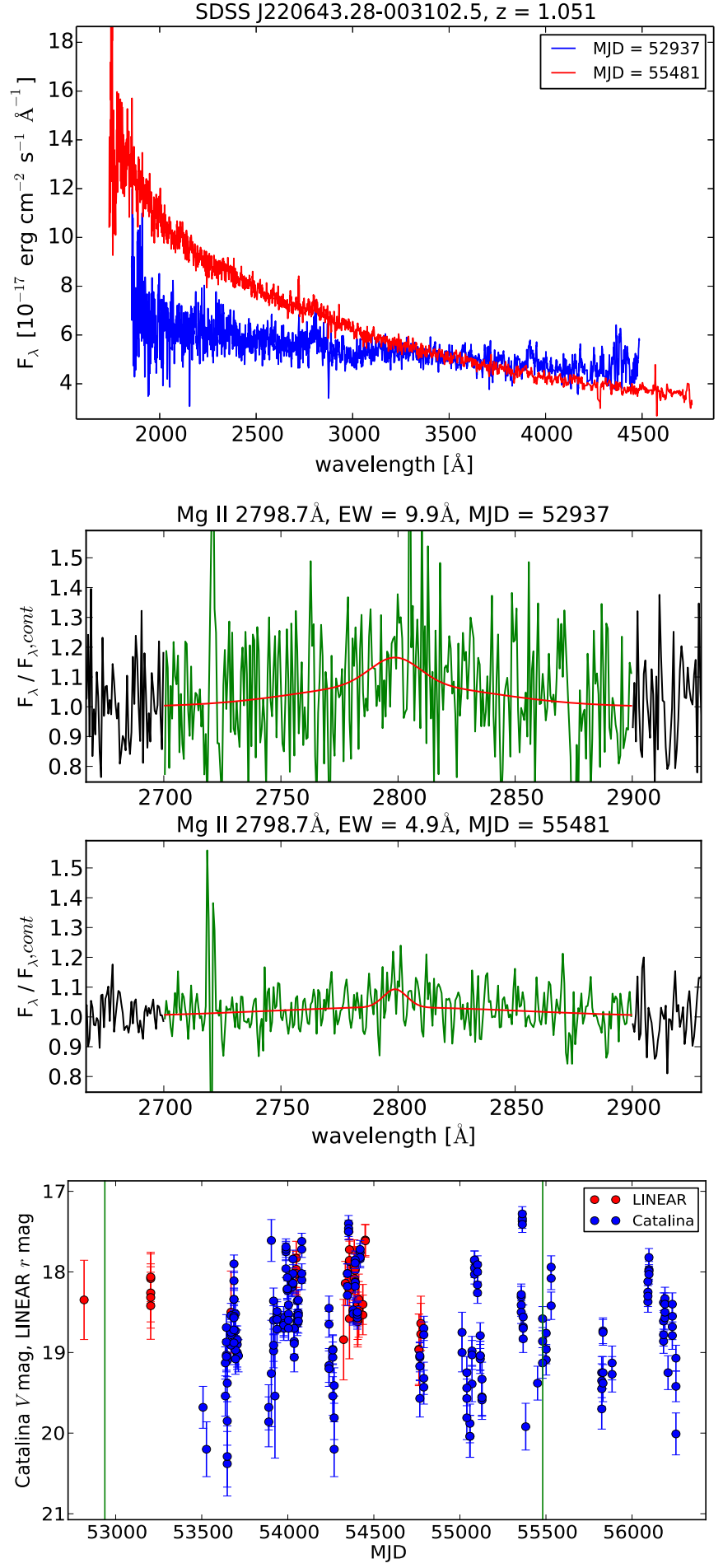

Figure 8. SDSS J220643.28-00312.5, similar to Figure 2.

(A color version of this figure is available in the online journal.)

characteristic timescale of variability of 6 days in the rest-frame is the shortest among the transition blazars in our sample. This blazar is in the Stripe 82 multi-epoch imaging portion of SDSS, and its SDSS optical light curve is among the most variable of all quasars in MacLeod et al. (2010).

\section{DISCUSSION}

In the previous sections, we explored the spectral, temporal, and multi-wavelength properties of our sample of transition 

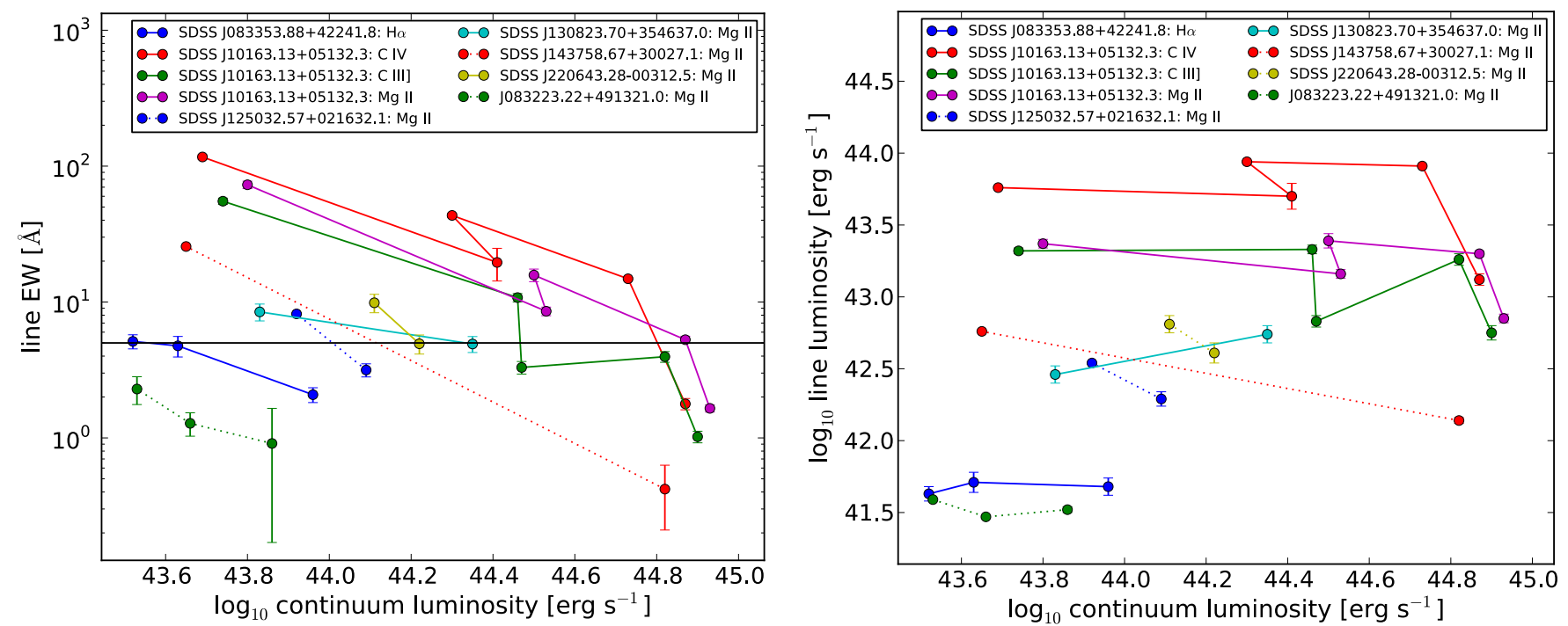

Figure 9. Left: broad emission line rest-frame equivalent width vs. the fitted continuum luminosity at the line for all lines present in the six transition blazars and one additional blazar in our sample. Measurements from all spectral epochs for each line are shown and connected in temporal order. The distinct anti-correlation strongly indicates that changes in the equivalent width of the lines are primarily due to swamping from the highly variable continuum. Right: similar to the left panel, but for the line luminosity vs. the continuum luminosity at the line. The line luminosities also show variability, possibly due to variability in the ionizing continuum from a radiatively efficient accretion disk.

(A color version of this figure is available in the online journal.)

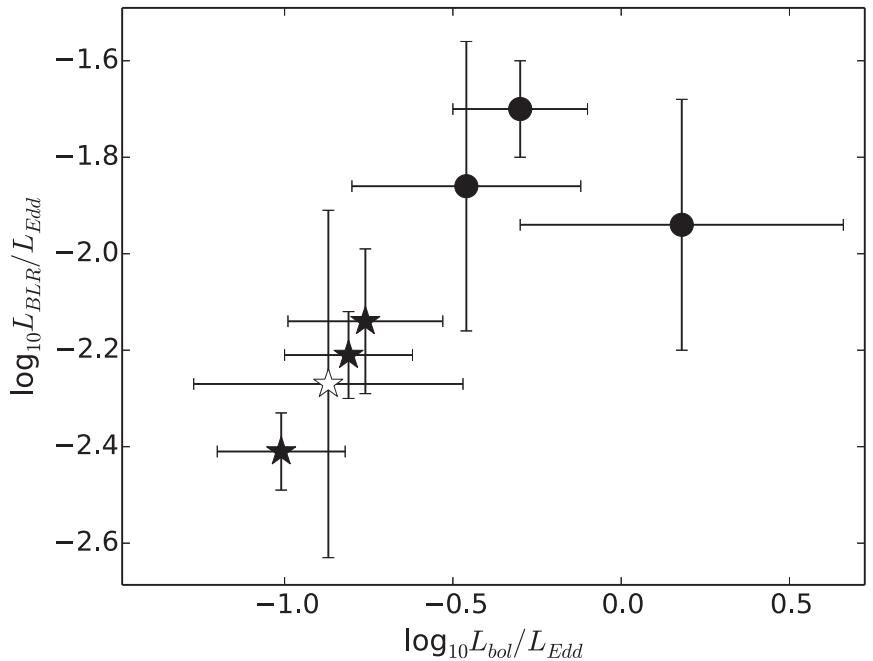

Figure 10. Bolometric Eddington ratio vs. the Eddington ratio of the total broad emission line luminosity for the six transition blazars in our sample (filled symbols) and the additional blazar that did not transition (open star). These values are listed in Table 1. Blazars with Fermi detections are shown with stars, and those without are shown with dots. These Eddington ratios are much larger than the $L_{\mathrm{bol}} / L_{\mathrm{Edd}} \sim 0.01$ typical of radiatively inefficient accretion flows in BLLs, and are instead consistent with those of classical thin accretion disks in FSRQs.

blazars. In this section, we will piece together these properties to understand the nature of these objects. The Eddington ratios of the transition blazars in our sample calculated in Section 3.1 (and shown in Figure 10) are more than an order of magnitude above the approximate $L_{\mathrm{bol}} / L_{\mathrm{Edd}} \sim 0.01$ value that typically separates the radiatively efficient and inefficient accretion regimes in AGNs, and are even higher than the $L_{\mathrm{bol}} / L_{\mathrm{Edd}} \sim 0.1$ typical of Type 1 quasars in SDSS (SH11). This result strongly suggests that the transition blazars in our sample are actually rapidly accreting FSRQs, which have been found to have systematically large bolometric Eddington ratios of $L_{\mathrm{bol}} / L_{\mathrm{Edd}} \sim 0.1-1$ in a large Fermi-selected sample (Shaw et al. 2012). The $L_{\mathrm{BLR}} /$
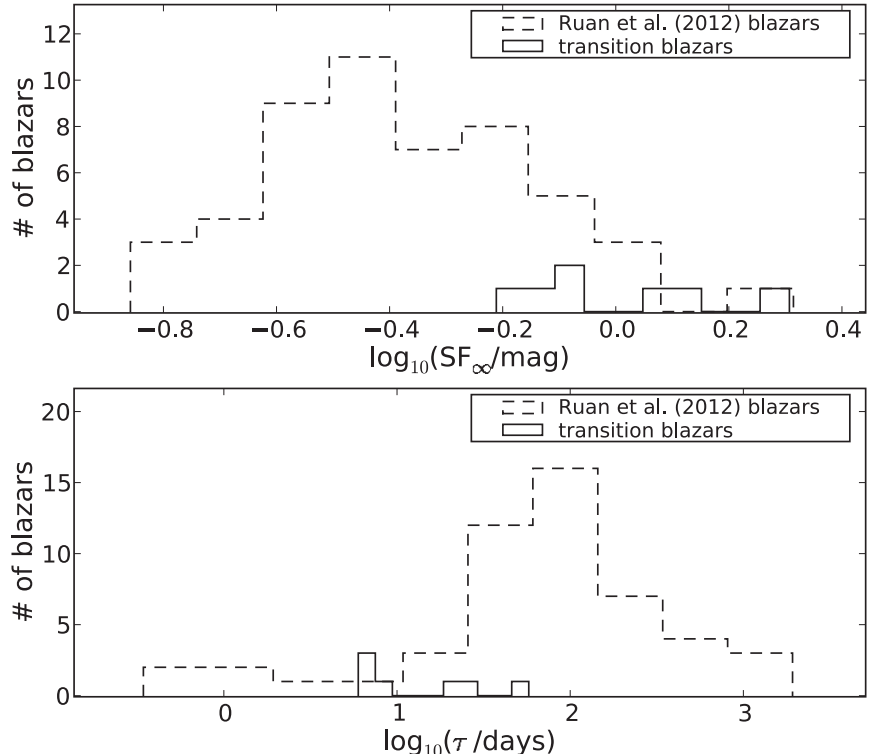

Figure 11. Top: histogram of the variability amplitude on long timescales for the long-term photometric light curves of the seven blazars in our sample modeled as a damped random-walk, as well as for a sample of 60 blazars from Ruan et al. (2012). Bottom: similar to the top panel, but for the characteristic timescale of variability in the rest-frame. The seven blazars in our sample have significantly larger variability amplitudes and shorter timescales of variability than typical blazars, supporting the interpretation that the jet emission is particularly strongly beamed.

$L_{\text {Edd }}$ of these transition blazars are also correspondingly well above the $\sim 5 \times 10^{-4}$ divide suggested by Ghisellini et al. (2011) to separate FSRQs from BLLs. We emphasize that our estimates of $L_{\mathrm{bol}} / L_{\mathrm{Edd}}$ for each transition blazar are not strongly affected by contamination from jet emission since the continuum luminosities (without jet contamination) are not directly measured in the spectrum, but rather indirectly estimated based on scaling relations with the BEL luminosities as discussed in Section 3.1. 
Our FSRQ-like interpretation of these blazars is further supported by the strong variability observed in the BEL luminosities of up to $\sim 0.5$ dex (Figure 9 , left panel). This effect is likely due to the strong flux variability of the ionizing thermal continuum from radiatively efficient thin accretion disks that are thought to exist in FSRQs, in contrast to the radiatively inefficient accretion flows in BLLs, since variations in the jet continuum only weakly correlate with BEL flux variability (Isler et al. 2013). The FSRQ nature of these transition blazars is also supported by our investigation of their SEDs (Section 3.2), which revealed that their synchrotron peaks are at frequencies of $\sim 10^{12-13} \mathrm{~Hz}$ and luminosities of $10^{45-46} \mathrm{erg} \mathrm{s}^{-1}$; these SED properties are similar to FSRQs and low synchrotron-peaked BLLs, although the underlying accretion state is difficult to discern from the SED alone.

Although swamping of BELs by the jet continuum in transition blazars as suggested by the observed anti-correlation between the line EWs and continuum luminosities (see Figure 9) may be expected in FSRQs, the results of our systematic search reveals that such cases are instead uncommon. Our investigation suggests that the transition phenomenon occurs in rare FSRQs with particularly strongly beamed jets oriented at extremely small angles along the line of sight. The particularly strong beaming in such objects would lead to a correspondingly strong jet dominance of the optical spectra, as well as strong flux variability. This interpretation is supported by our investigation of the long-term optical variability of these transition blazars in Section 3.4: they show larger amplitudes and shorter timescales of variability (Figure 11) than typical blazars. This uniquely strong variability in transition blazars suggests that the jet beaming is especially strong in these objects, with large Lorentz factors.

The transitional nature of the BEL EWs in these blazars may lead to misclassifications if the subclass classification is based solely on EWs in single-epoch spectra as traditionally done. Although transition blazars are rare, misclassification can still significantly affect investigations of blazar redshift evolution at high redshifts due to their rarity. Our findings suggest that some BLLs may actually be strongly beamed FSRQs, thus decreasing the apparent BLL population, especially at the higher redshifts of FSRQs and partially due to the high luminosities of these particularly strongly beamed blazars. This effect can decrease the small sample sizes of high-redshift BLLs used in investigations of their redshift evolution, and may even add credence to claims of a BLL negative redshift evolution. Since the SEDs of the transition blazars in our sample are ambiguous between FSRQs and low synchrotron-peaked BLLs despite their unambiguously large accretion rates, classification based on SEDs can also lead to incorrect results. Even when multiepoch spectra are available (thus allowing for identification of transition blazars), some studies have incorrectly assumed that transitional blazars in which all BELs have EW $<5 \AA$ in any epoch are BLLs (e.g., Shaw et al. 2012, 2013b). In contrast, our investigation reveals that these transition blazars are instead likely to be FSRQs.

Similar to the arguments presented in Ghisellini et al. (2011), we strongly recommend that classification of ambiguous blazars be based on Eddington ratios, especially through the use of multi-epoch spectra so that the BEL properties can be robustly determined from the large-EW spectral epochs. This Eddingtonratio-based classification is useful not only for transition blazars, but also for blazars such as SDSS 083223.22+491321.0 (Figure 8), which does not have BEL EW $>5 \AA$ in any of the observed epochs. Although this blazar appears to be a canonical BLL, its Eddington ratio of $L_{\text {bol }} / L_{\text {Edd }} \sim 0.17$ instead clearly reveals it to be an FSRQ with extreme swamping of the BELs. Additionally, the large EW variations in transition blazars observed in multi-epoch spectroscopy also aids robust redshift determinations.

Finally, despite the strong beaming in their jets that cause strong photometric variability and EW changes in the BELs, it is puzzling that not all of these transition blazars are $\gamma$-ray detected. The synchrotron peak frequencies and luminosities in SEDs of the transition blazars in our sample are all in a similar range, without any clear distinction between the $\gamma$-ray loud and quiet blazars. It is possible that the $\gamma$-ray luminosity in these jets with extreme beaming may be suppressed due to strong $\gamma \gamma$ attenuation from pair production (Becker \& Kafatos 1995; Blandford \& Levinson 1995) or Klein-Nishina effects in the electron Compton cooling (e.g., Moderski et al. 2005). Many of the transition blazars in our sample have detections in GALEX, Swift, and ROSAT in addition to Fermi; future investigations of the high-energy inverse-Compton SED peaks of these blazars will be able to shed light on this problem. Future studies of $\gamma$ ray variability in these transition blazars will also be able probe the physical mechanism of transitions between jet-dominated and disk-dominated states (Chatterjee et al. 2013).

\section{CONCLUSIONS}

Using a large sample of 602 pairs of repeat blazar spectra of 354 blazars from SDSS, we present a systematic search for blazars that have transitioned between the BLL and FSRQ subclasses based on the EW of the BELs. These repeat spectral observations span timescales from intra-night to $\sim 12.5 \mathrm{yr}$ in the observed-frame, and are selected by a variety of multiwavelength properties. We find six clear cases of transition blazars that show dramatic variability in their BEL EWs; the strong BEL EW variability allows us to determine new redshifts for two of the transition blazars in our sample, as well as one additional blazar. We investigate the SDSS spectral, $\gamma$-ray, SED, and optical variability properties of these transition blazars to understand their nature as probed by these methods. Our key results are as follows.

1. EWs of BELs in transition blazars can vary dramatically, with observed variations up to factors of $>60$. Transition blazars have high accretion rates with bolometric Eddington ratios typical of FSRQs. A clear anti-correlation between the continuum luminosity and BEL EWs strongly suggests that the transitional phenomenon is primarily due to swamping of the BELs by the highly variable jet continuum. However, the luminosities of the BELs are also variable, further supporting the existence of radiatively efficient thin accretion disks in these objects.

2. The swamping of the BELs in transition blazars occurs when the jet continuum is strongly beamed, placing transition blazars as rare cases of FSRQs with particularly strongly beamed jets. This interpretation is supported by analysis of their long-term photometric light curves, which display larger amplitudes and shorter timescales of variability than typical blazars, consistent with the effects of strongly beamed jet continuum emission.

3. The SEDs of these transition blazars show low-frequency, luminous synchrotron peaks, similar to FSRQs and lowpeaked BLLs. Their $\gamma$-ray properties (where detected) are also ambiguous between these two classes. Since 
classification based on BEL EWs, SEDs, and $\gamma$-ray properties of these objects is difficult, we suggest the use of Eddington ratios.

Although transition blazars are rare, correct classification will impact studies of the divergent properties of BLLs and FSRQs, especially regarding their redshift evolution. Future directions for investigation of these rare transition blazars can focus on understanding the nature of the strong beaming using radio observations, and study of their high-energy inverse-Compton SED peaks to understand why only some of these strongly beamed FSRQs are $\gamma$-ray loud. Future surveys, such as the Time-Domain Spectroscopic Survey (Green et al. 2014) of SDSS-IV, will include more repeat spectra of blazars and will uncover many more such transitional cases.

J.J.R. thanks James R. A. Davenport for helpful discussions. Support for J.J.R. was provided by NASA through Chandra Award Numbers AR9-0015X, AR0-11014X, and AR2-13007X, issued by the Chandra X-ray Observatory Center, which is operated by the Smithsonian Astrophysical Observatory for and on behalf of NASA under contract NAS8-03060. Support for W.N.B. was provided by NSF grant AST-1108604 and NASA ADP grant NNX10AC99G.

Funding for SDSS-III has been provided by the Alfred P. Sloan Foundation, the Participating Institutions, the National Science Foundation, and the U.S. Department of Energy Office of Science. The SDSS-III Web site is http://www.sdss3.org/.

SDSS-III is managed by the Astrophysical Research Consortium for the Participating Institutions of the SDSS-III Collaboration including the University of Arizona, the Brazilian Participation Group, Brookhaven National Laboratory, Carnegie Mellon University, University of Florida, the French Participation Group, the German Participation Group, Harvard University, the Instituto de Astrofisica de Canarias, the Michigan State/ Notre Dame/JINA Participation Group, Johns Hopkins University, Lawrence Berkeley National Laboratory, Max Planck Institute for Astrophysics, Max Planck Institute for Extraterrestrial Physics, New Mexico State University, New York University, Ohio State University, Pennsylvania State University, University of Portsmouth, Princeton University, the Spanish Participation Group, University of Tokyo, University of Utah, Vanderbilt University, University of Virginia, University of Washington, and Yale University.

The LINEAR program is sponsored by the National Aeronautics and Space Administration (NRA No. NNH09ZDA001N, 09-NEOO09-0010) and the United States Air Force under Air Force Contract FA8721-05-C-0002. Opinions, interpretations, conclusions, and recommendations are those of the authors and are not necessarily endorsed by the United States Government.

The Catalina Sky Survey is funded by the National Aeronautics and Space Administration under grant No. NNG05GF22G issued through the Science Mission Directorate Near-Earth Objects Observations Program. The CRTS survey is supported by the U.S. National Science Foundation under grants AST0909182.

Part of this work is based on archival data, software, or online services provided by the ASI Science Data Center (ASDC).

\section{REFERENCES}

Abdo, A. A., Ackermann, M., Agudo, I., et al. 2010a, ApJ, 716, 30 Abdo, A. A., Ackermann, M., Ajello, M., et al. 2010b, ApJ, 720, 435 Ackermann, M., Ajello, M., Allafort, A., et al. 2011, ApJ, 743, 171 Ahn, C. P., Alexandroff, R., Allende Prieto, C., et al. 2014, ApJS, 211, 17
Antonucci, R. 1993, ARA\&A, 31, 473

Atwood, W. B., Abdo, A. A., Ackermann, M., et al. 2009, ApJ, 697, 1071

Bauer, A., Baltay, C., Coppi, P., et al. 2009, ApJ, 705, 46

Becker, P. A., \& Kafatos, M. 1995, ApJ, 453, 83

Beckmann, V., Engels, D., Bade, N., \& Wucknitz, O. 2003, A\&A, 401, 927

Blandford, R. D., \& Levinson, A. 1995, ApJ, 441, 79

Blandford, R. D., \& Rees, M. J. 1978, in Proc. Pittsburgh Conf. on BL Lac Objects (Pittsburgh, PA: Univ. Pittsburgh), 328

Bolton, A. S., Schlegel, D. J., Aubourg, É., et al. 2012, AJ, 144, 144

Böttcher, M., \& Dermer, C. D. 2002, ApJ, 564, 86

Caccianiga, A., Maccacaro, T., Wolter, A., Della Ceca, R., \& Gioia, I. M. 2002, ApJ, 566, 181

Cardelli, J. A., Clayton, G. C., \& Mathis, J. S. 1989, ApJ, 345, 245

Celotti, A., Padovani, P., \& Ghisellini, G. 1997, MNRAS, 286, 415

Chatterjee, R., Nalewajko, K., \& Myers, A. D. 2013, ApJL, 771, L25

Corbett, E. A., Robinson, A., Axon, D. J., et al. 1996, MNRAS, 281, 737

Corbett, E. A., Robinson, A., Axon, D. J., \& Hough, J. H. 2000, MNRAS, 311,485

Dai, H., Xie, G. Z., Zhou, S. B., et al. 2007, AJ, 133, 2187

Dawson, K. S., Schlegel, D. J., Ahn, C. P., et al. 2013, AJ, 145, 10

Drake, A. J., Djorgovski, S. G., Mahabal, A., et al. 2009, ApJ, 696, 870

Eisenstein, D. J., Weinberg, D. H., Agol, E., et al. 2011, AJ, 142, 72

Fine, S., Jarvis, M. J., \& Mauch, T. 2011, MNRAS, 412, 213

Fossati, G., Maraschi, L., Celotti, A., Comastri, A., \& Ghisellini, G. 1998, MNRAS, 299, 433

Francis, P. J., Hewett, P. C., Foltz, C. B., et al. 1991, ApJ, 373, 465

Gaskell, C. M. 1982, ApJ, 263, 79

Ghisellini, G., \& Tavecchio, F. 2008, MNRAS, 387, 1669

Ghisellini, G., Tavecchio, F., Foschini, L., \& Ghirlanda, G. 2011, MNRAS, 414, 2674

Giommi, P., Padovani, P., Polenta, G., et al. 2012, MNRAS, 420, 2899

Green, P. J., Anderson, S. F., Morganson, E., et al. 2014, BAAS, 223, 116.15

Greene, J. E., \& Ho, L. C. 2005, ApJ, 630, 122

Gunn, J. E., Siegmund, W. A., Mannery, E. J., et al. 2006, AJ, 131, 2332

Hinshaw, G., Larson, D., Komatsu, E., et al. 2013, ApJS, 208, 19

Isler, J. C., Urry, C. M., Coppi, P., et al. 2013, ApJ, 779, 100

Jarvis, M. J., \& McLure, R. J. 2006, MNRAS, 369, 182

Kozłowski, S., Kochanek, C. S., Udalski, A., et al. 2010, ApJ, 708, 927

Maccacaro, T., Gioia, I. M., Maccagni, D., \& Stocke, J. T. 1984, ApJL, 284, L23

MacLeod, C. L., Ivezić, Ž., Kochanek, C. S., et al. 2010, ApJ, 721, 1014

Maraschi, L., \& Tavecchio, F. 2003, ApJ, 593, 667

Marchã, M. J. M., \& Caccianiga, A. 2013, MNRAS, 430, 2464

Massaro, E., Giommi, P., Leto, C., et al. 2009, A\&A, 495, 691

McLure, R. J., \& Dunlop, J. S. 2004, MNRAS, 352, 1390

Moderski, R., Sikora, M., Coppi, P. S., \& Aharonian, F. 2005, MNRAS, 363,954

Narayan, R., \& Yi, I. 1994, ApJL, 428, L13

Nolan, P. L., Abdo, A. A., Ackermann, M., et al. 2012, ApJS, 199, 31

Padovani, P., \& Giommi, P. 1995, MNRAS, 277, 1477

Padovani, P., Giommi, P., Landt, H., \& Perlman, E. S. 2007, ApJ, 662, 182

Pâris, I., Petitjean, P., Aubourg, É., et al. 2014, A\&A, 563, A54

Perlman, E. S., Stocke, J. T., Schachter, J. F., et al. 1996, ApJS, 104, 251

Plotkin, R. M., Anderson, S. F., Brandt, W. N., et al. 2010, AJ, 139, 390

Rector, T. A., Stocke, J. T., Perlman, E. S., Morris, S. L., \& Gioia, I. M. 2000, AJ, 120,1626

Richards, G. T., Kruczek, N. E., Gallagher, S. C., et al. 2011, AJ, 141, 167

Richards, G. T., Lacy, M., Storrie-Lombardi, L. J., et al. 2006, ApJS, 166, 470

Richards, G. T., Vanden Berk, D. E., Reichard, T. A., et al. 2002, AJ, 124, 1

Ross, N. P., Myers, A. D., Sheldon, E. S., et al. 2012, ApJS, 199, 3

Ruan, J. J., Anderson, S. F., MacLeod, C. L., et al. 2012, ApJ, 760, 51

Runnoe, J. C., Brotherton, M. S., Shang, Z., Wills, B. J., \& DiPompeo, M. A. 2013, MNRAS, 429, 135

Sambruna, R. M., Maraschi, L., \& Urry, C. M. 1996, ApJ, 463, 444

Sbarrato, T., Ghisellini, G., Maraschi, L., \& Colpi, M. 2012, MNRAS, 421,1764

Schlegel, D. J., Finkbeiner, D. P., \& Davis, M. 1998, ApJ, 500, 525

Sesar, B., Stuart, J. S., Ivezić, Ž., et al. 2011, AJ, 142, 190

Shaw, M. S., Filippenko, A. V., Romani, R. W., Cenko, S. B., \& Li, W. 2013a, AJ, 142,127

Shaw, M. S., Romani, R. W., Cotter, G., et al. 2012, ApJ, 748, 49

Shaw, M. S., Romani, R. W., Cotter, G., et al. 2013b, ApJ, 764, 135

Shen, Y., Greene, J. E., Strauss, M. A., Richards, G. T., \& Schneider, D. P. 2008, ApJ, 680, 169

Shen, Y., Richards, G. T., Strauss, M. A., et al. 2011, ApJS, 194, 45

Smee, S. A., Gunn, J. E., Uomoto, A., et al. 2013, AJ, 146, 32 
Stickel, M., Padovani, P., Urry, C. M., Fried, J. W., \& Kuehr, H. 1991, ApJ, 374,431

Stocke, J. T., Morris, S. L., Gioia, I. M., et al. 1991, ApJS, 76, 813

Stokes, G. H., Evans, J. B., Viggh, H. E. M., Shelly, F. C., \& Pearce, E. C. 2000, Icar, 148, 21

Stoughton, C., Lupton, R. H., Bernardi, M., et al. 2002, AJ, 123, 485

Stratta, G., Capalbi, M., Giommi, P., et al. 2011, arXiv:1103.0749
Urry, C. M., \& Padovani, P. 1995, PASP, 107, 803

Vanden Berk, D. E., Richards, G. T., Bauer, A., et al. 2001, AJ, 122, 549

Vermeulen, R. C., Ogle, P. M., Tran, H. D., et al. 1995, ApJL, 452, L5

Wang, J.-M., Staubert, R., \& Ho, L. C. 2002, ApJ, 579, 554

Wills, B. J., \& Browne, I. W. A. 1986, ApJ, 302, 56

Wolter, A., Caccianiga, A., della Ceca, R., \& Maccacaro, T. 1994, ApJ, 433, 29

York, D. G., Adelman, J., Anderson, J. E., Jr., et al. 2000, AJ, 120, 1579 